\title{
Editorial
}

Angela Duparré*

\section{Topical issue on optical surfaces}

https://doi.org/10.1515/aot-2017-0058

'God made the bulk; the surface was invented by the devil' is a widely known quotation attributed to the 1945 Nobel Laureate Wolfgang Pauli. Even though this remark was then meant to refer to surface properties on the atomic scale in a surface science context, it holds for larger scales and modern optical application aspects as well: Optical surfaces as fundamental parts in optical systems are facing ever-increasing and highly complex demands from industry and research. This is driven by tough requirements for high quality, cost and time reduction in production and research and development, and by rapidly growing and new application fields. Large optical surfaces with arbitrary shapes and the enormous progress of nanotechnologies are just two examples. These challenging developments have led to substantially improved, novel, and very sophisticated optical design, fabrication, and measurement techniques and have also influenced the way these tools are deployed. Their variety, interdependence and the diversity of required scientific and technological know-how can indeed make optical surface development ‘devilish' tricky.

The diverse disciplines encompassed by the term Optical Surface - such as optical surface simulation and design, manufacturing processes (grinding, polishing, etc.) coating technologies (including thin film design), functionalization (adjusted wetting properties, etc.), contamination control, and characterization and metrology - have each established their particular scientific communities, international conferences and specific books. Naturally, this makes it impossible to be covered extensively in just one topical issue of a journal. But what we try to do is to provide just a few examples out of these manifold areas, which we hope illustrate the latest achievements as well as the complexity of optical surface research and development. Papers for this issue were solicited from individuals and groups who have been doing exciting work in the field.

*Corresponding author: Angela Duparré, Surface and Thin Film Consultant/Fraunhofer IOF, Jena, Germany, e-mail: angela.duparre@iof.fraunhofer.de

www.degruyter.com/aot

(c) 2017 THOSS Media and De Gruyter
We start with two papers on optical design for freeform surfaces by Broemel et al., which in part one provide the mathematical basis, followed by considerations of practical application aspects in part two. They can, however, be read independently of each other as well, according to the individual focus of interests of you, the audience.

An example of optical fabrication is given in the article by Faehnle et al., which discusses alternative approaches of grinding procedures for brittle materials. Besides the essential manufacturing details, it reveals the importance of surface characterization to correlate process parameters and surface quality.

The material aspect and the substantial role of measurement techniques are also addressed in the paper by Schroeder et al., here with respect to laser induced damage of optical surfaces including thin film coatings. It presents a new damage testing method and its combination with in-situ light scattering analysis.

The next paper, by v. Finck et al., considers light scattering from optical surfaces as a critical optical loss that has to be reduced as much as possible. In this article we meet the complex interaction of design (this time for optical coatings), surface contamination and light scattering characterization.

Light scattering remains the topic of the article by Kroneberger as well. In the context of diffraction gratings, it discusses the often underestimated problem of correlating light scattering simulation and modeling of optical surfaces with appropriate measurement data.

The collection of papers concludes with the contribution by Felde et al., which is dedicated to optical surfaces with additional functional effects, in particular hydrophobic and hydrophilic properties. Again, the connection of different surface aspects determines the outcome, here with respect to thin film coatings and the combination of wetting, roughness and light scatter characterization.

Finally, I would like to thank the invited authors for their valued contributions, the Editor-in-Chief and the publisher for the opportunity to put this topical issue together, and the Advanced Optical Technologies team and the reviewers for their support in its realization.

Happy reading

Angela Duparré 


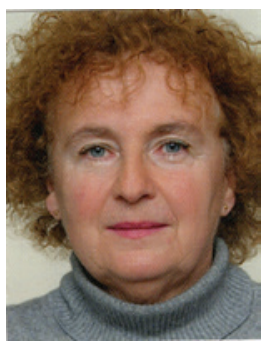

Angela Duparré

Surface and Thin Film Consultant/Fraunhofer

IOF, Jena, Germany

angela.duparre@iof.fraunhofer.de

Angela Duparré received her PhD degree from the Physics Faculty of the Friedrich Schiller University (FSU) of Jena in 1985. From 1985 to 1992 she pursued research on optical surfaces and thin films at the FSU and taught physics courses to students. In 1988 she was a visiting scientist at the Lebedev Physical Institute in Moscow and in 1991 at CREOL, University of Central Florida in Orlando. In 1992 she joined the newly founded Fraunhofer Institute for Applied Optics and Precision Engineering in Jena to become head of the Surface and Thin Film Characterization Group. Her interests have since been directed to the study of optical surface and thin film properties such as light scattering, nanostructures, roughness and functional effects. She has published more than 150 papers and is involved in the organization of conferences on optical surface metrology, fabrication and coatings. In 2011, she was elected a fellow of the European Optical Society. Recently retired, she now continues her activities in the field as a consultant. 